\title{
Experiences with GeneRecon on MiG
}

\author{
Thomas Mailund $^{\mathrm{a}}$, Christian N.S. Pedersen ${ }^{\mathrm{a}, \mathrm{b}}$, Jonas Bardino ${ }^{\mathrm{c}, *}$, Brian Vinter $^{\mathrm{c}}$, Henrik H. Karlsen ${ }^{\mathrm{c}}$ \\ ${ }^{a}$ Bioinformatics Research Center, University of Aarhus, Denmark \\ ${ }^{\mathrm{b}}$ Department of Computer Science, University of Aarhus, Denmark \\ ${ }^{\mathrm{c}}$ Department of Computer Science, University of Copenhagen, Denmark
}

Received 28 February 2006; received in revised form 14 July 2006; accepted 16 September 2006

Available online 14 November 2006

\begin{abstract}
We report on our experiences so far with running a bioinformatics simulation study on a newly developed Grid architecture. We briefly describe the bioinformatics application - an association mapping algorithm for both locating disease loci and separating cases into those diseased due to genetic factors and those diseased solely due to environment factors - and describe the Grid architecture and how the application is set up to run on the Grid.
\end{abstract}

(C) 2006 Elsevier B.V. All rights reserved.

Keywords: Minimum intrusion Grid; Association mapping; Simulation study

\section{Introduction}

Locating the genetic factors behind common inheritable diseases is a highly relevant problem in medicine and bioinformatics [3]. The complexity of the problem, however, implies that finding biologically sound solutions requires algorithms of high time complexity, which makes extensive experimentation difficult. This paper describes the work done, and results from, utilising Grid computing [1] for large scale experiments with an algorithm, GeneRecon, for detecting genetic mutation clusters.

GeneRecon is a tool for analysis of population genetic data from case/control studies. The analysis works on genetic data which is collected from patients with a specific disease and a control group without this disease, and tries to locate genes that are increasing the risk of disease. The analysis, which is based on a Markov-chain Monte Carlo (MCMC) method, is extremely CPU-demanding, since it searches through a very large parameter-space. The presented work seeks to evaluate a method that both significantly reduces the running time of the method and additionally can determine which patients have a

\footnotetext{
* Corresponding author.

E-mail addresses: mailund@birc.au.dk (T. Mailund), cstorm@birc.au.dk (C.N.S. Pedersen), bardino@diku.dk (J. Bardino), vinter@diku.dk (B. Vinter), karlsen@diku.dk (H.H. Karlsen).
}

given disease because of a genetic markup, rather than another cause, e.g. environmental effects. The work includes thousands of GeneRecon runs - an infeasible task without access to a large number of processors.

Minimum intrusion Grid (MiG) [10] is a new platform for Grid computing which is driven by a stand-alone approach to Grid, rather than integration with existing systems. The goal of the MiG project is to provide a Grid infrastructure where the requirements on users and resources alike, to join Grid, is as small as possible. While striving for minimum intrusion, MiG will still seek to provide a feature-rich and dependable Grid solution.

\section{Association mapping and mutation clusters}

For diseases with a genetic component, carriers of high risk alleles are often descendants of one or a few founders, in whose genome the allele appeared by mutation. Recent shared ancestry of the carriers in the disease position results in a higher homogeneity, among the carriers, in the genomic region around the disease affecting gene, than in the population at large. This, in turn, causes markers - points of genetic variation - around the disease gene to be more associated with each other - in a statistical sense, a phenomenon known as linkage disequilibrium, or LD. The use of LD for disease gene mapping has in recent years received much attention, and is believed by 
many to hold great promise in the mapping of common complex diseases [3].

The Common Disease, Common Variant (CD/CV) hypothesis [9], fundamental to association mapping and based on the history of the human population, states that most common diseases with a genetic component are affected by a few alleles with a (relatively) high frequency in the population but with a (relatively) low penetrance. If it holds true, we would expect that, in a case/control study, several unaffected individuals would be carriers of the increased risk mutation - but not diseased, due to the low penetrance - and perhaps, at the same time, only a few of the diseased individuals would carry the increased risk mutation while the remaining affected individuals have the disease due to environmental causes. In such a study, identifying the disease carriers among the affected individuals would greatly benefit the search for the disease gene once a candidate genome region has been found.

\subsection{The mapping method based on mutation and null clusters}

To approach this problem, we have developed an MCMC algorithm ${ }^{1}$ based on Morris et al.'s shattered coalescent method [8], but incorporating ideas from Liu et al. [4] and Molitor et al. [7], where we separated the affected individuals into mutation clusters - where affected individuals in the same cluster are assumed to be descendants of a common founder - and a null cluster - for individuals affected due to environmental factors and not genetic factors. By sampling, during the run of the MCMC, the distribution of affected individuals among the mutation and null clusters, we hope to be able to infer which individuals carry an increased risk of mutation and which are affected solely due to environmental factors, while at the same time locating the locus of the disease affecting gene. Introducing the mutation and null cluster can potentially also greatly reduce the running time of the algorithm, as we only explicitly model the genealogy of the cases in the mutation clusters, not of all cases, thus reducing the search space significantly.

While the methods we have based our new technique upon have proved themselves highly efficient in locating the disease locus on case/control data, they are unfortunately also very CPU-demanding and require several hours - or days - for a successful computation with a few hundreds of cases and controls, with a few tens of markers. This makes computer clusters or computer Grid architectures essential for validating new mapping methods such as ours, since such validation requires analysis of a large number of (simulated) data sets to get useful statistics about the performance of the method.

\subsection{The experimental setup}

We have simulated haplotype data sets using the CoaSim simulator [5] under varying recombination rate $(\rho=40$ and $\rho=400$, roughly corresponding to $0.1 \mathrm{cM}$ and $1 \mathrm{cM}$ ), with

\footnotetext{
1 The new MCMC algorithm has been implemented in the association mapping tool GeneRecon (http://www.daimi.au.dk/rmailund/GeneRecon) [6].
}

varying marker densities (20 markers on the region, 40 markers on the region, and a twice as wide region with 40 markers, 20 of which are in the middle region - containing the disease marker - and 10 on each side), and varying disease models ( $m / w$ where $m$ is the fraction of affected individuals among the mutants and $w$ is the fraction of affected individuals among the wild types).

For each data set, four chains of GeneRecon was run for cluster sizes 100 (all affected individuals), 75, and 50. The analysis of the results was conducted using a set of $R$ scripts. In calculating the error of disease locus inference, we have used a simple measure of the distance from the point with maximum posterior value to the true disease locus. This does not catch the confidence of the inference, but gives a simple measure of error that lets us compare the accuracy for various cluster sizes.

\section{Minimum intrusion Grid}

MiG (http://www.migrid.org) is a Grid middleware model and implementation designed with previous Grid middleware experiences in mind. In MiG central issues such as security, scalability, privacy, strong scheduling and fault tolerance are included by design. Other Grid middlewares tend to suffer from problems with one or more of those issues.

The MiG model seeks to be non-intrusive in the sense that both users and resources should be able to join the Grid with a minimal initial effort and with little or no maintenance required. One way to obtain these features is keeping the required software installation to a functional minimum, e.g. the software that is required to run MiG includes only 'need to have' features, while any 'nice to have' features are completely optional. This design philosophy has been used, and reiterated, so stringently that in fact neither users nor resources are required to install any software that is MiG specific.

Another area where MiG strives to be non-intrusive is the communication with users and resources. Users in general and resources in particular cannot be expected to have unrestricted network access in either direction. Therefore the MiG design enforces that all communication with resources and users should use only the most common protocols known to be allowed even with severely restricted networking configurations. Furthermore resources should neither be forced to provide any special privileges to the MiG job execution user(s) nor run any additional network-listening daemons.

Fig. 1 depicts the way MiG separates the users and resources with a physical Grid layer, which users and resources securely access through one of a number of MiG servers. The MiG model resembles a classic client-server model where clients are represented by either users or resources. The servers are represented by the Grid itself, which in the case of MiG is a set of actual computers, not simply a protocol for communicating between computers. All clients can contact the Grid in order to either upload or download files. User clients can additionally contact the server in order to do job management and file manipulation while resource clients can additionally request a job to execute. As much of the actual functionality as possible is located at the MiG servers, where it is maintained by the MiG developers. Thus, in addition to minimising the user 


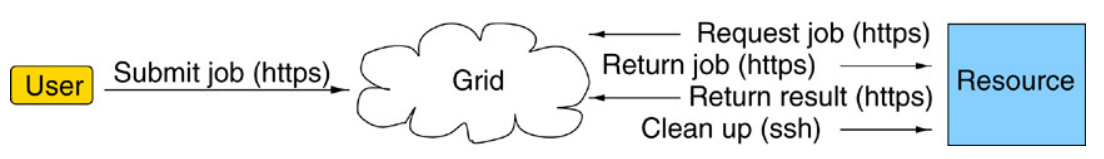

Fig. 1. MiG: Architectural overview.

and resource requirements, the Grid layer simplifies consistent deployment of software upgrades.

The security infrastructure relies on all entities: users, $\mathrm{MiG}$ servers and resources, being identified by a signed certificate and a private key. The security model is session based and thus requires no insecure transfers or storage of certificates or proxycertificates contrary to a number of other Grid middlewares. Users communicate securely with the server by means of the HTTPS protocol using certificates for two-sided authentication and authorisation. Server communication with the resources is slightly more complicated as it combines SSH and HTTPS communication to provide secure communication along with the ability to remotely clean up after job executions.

MiG jobs are described with minimal Resource Specification Language (mRSL), which sticks with the philosophy of minimum intrusion, by hiding as many aspects of Grid computing as possible from the user. The same philosophy applies to storage in the way that MiG supplies every user with a Grid home directory where input and output files are stored. When a job references an input or output file, the location is simply given relative to the user's home directory and thus all aspects of storage elements and transfer protocols are completely hidden from the user. The user can access her home directory through a web interface or through a set of simple MiG executables for use with scripting.

Users simply submit jobs to the MiG server, which in turn handles everything from scheduling and job handout with input and output file management to notification upon job completion. An important aspect of this is that a job is not scheduled to a resource before the resource is ready to execute the job. Resources request jobs from the MiG server when they become ready. The MiG server subsequently seeks to schedule a suitable job for execution at the resource. If one is found, the job with input files is immediately handed out to the resource. Otherwise the resource is told to re-request a job later. Upon completion of a job, the resource hands the result back to the MiG server which then makes the result available to the user through her home directory and sends out any requested notifications.

MiG supports cooperation between users in virtual organizations through the VGrid [2] concept. In that way the members of a virtual organization can access shared files and resources just as easily as they would access their own files and public resources.

Even though MiG is a new model, we have already implemented a stable single server version. It relies on the Apache web server (http://httpd.apache.org/) as a basis for the web interface and further functionality is handled by a number of cgi scripts communicating with a local MiG server process. We have decided to implement as much of the project as possible in Python (http://www.python.org/) since it provides a very clear syntax, a high level of abstraction and it allows rapid development. The test MiG server used for the Mutation Clusters experiment has three permanent resource sites associated: two clusters and a Network of Workstations. All in all those resources deliver the computational power of about 128 Pentium 4 CPUs at all times.

\section{Mutation clusters on MiG}

Since the Mutation Clusters experiment is basically a large parameter sweep, the entire experiment can easily be partitioned into a lot of similar, independent jobs, where each job can be done in a reasonable amount of time - ranging from hours to about a week - on a standard single-CPU computer. This makes the experiment setup very simple, as each job can be sent to almost any available resource, and any failed jobs can just be re-executed at a later time or on another resource. Parallelism comes from different jobs being executed concurrently on separate resources rather than parallelism within each job. Thus the parallel execution of the Mutation Clusters experiment can be expected to be highly efficient.

\subsection{Running GeneRecon on MiG}

Grid execution of an application generally requires some preparations. Apart from creating the Grid-specific job description most of the preparations are of a general kind that would apply to any Grid or batch system. To maximise the number of suitable resources for the Grid jobs, it is necessary to minimise external dependencies. In this case the original version of the application depended on the availability of Guile Scheme (http://www.gnu.org/software/guile/), which resources in general cannot be expected to provide, thus we chose to statically link it into the software. Then the executable only depended on having a suitable $\mathrm{C}$ library available at the resources.

Since we already knew how to manually run the jobs from the command line, creating a job description was mostly a matter of filling in the command line information in an mRSL template. Output files that would normally have to be fetched from the resource in a manual setup were specified as output files in the mRSL to make them automatically available in the MiG home directory after job execution.

It turned out that the three main resource sites provided different versions of the $\mathrm{C}$ library, so although the software 


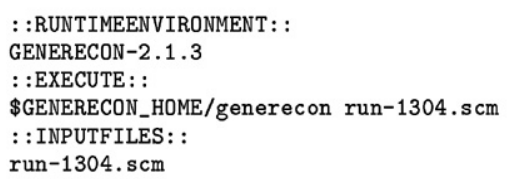

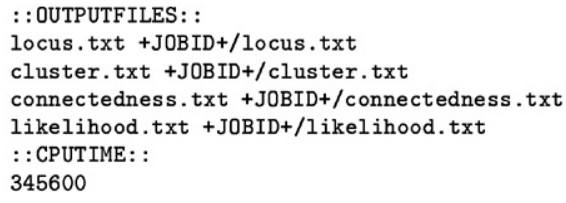

Fig. 2. An mRSL specification for a mutation cluster run (split in two columns here to save space).

could be compiled on all the platforms, we needed different versions for each. Thus we created and deployed a runtime environment to be able to select the right version for job execution on a particular resource. Strictly speaking jobs cannot expect any tools, libraries or devices to be generally available at resources. A runtime environment can be seen as a contract between users and resources - it describes a particular execution environment that jobs can expect to be available and that resources guarantee. When a job specifies a required runtime environment in the job description, the job will only be scheduled on a resource which advertises that runtime environment. Any actions required to provide that particular environment will automatically be taken during the job handout to the resource, so that it is available for job execution. The path to the correct version of the compiled tool was then simply selected by specifying the environment variable GENERECON_HOME.

The mRSL example in Fig. 2 shouldn't be hard to understand. The : : RUNTIMEENVIRONMENT : : section specifies that we wish to use a resource that offers the GeneRecon runtime-environment. The : :EXECUTE: : block holds the commands to be run in the job, which is only generecon in our case, the path to generecon is guaranteed to be \$GENERECON_HOME since we have required the GeneRecon runtime-environment. The generecon binary should be run with the parameter run-1304. scm which is also listed under $:$ : INPUTFILES : :, meaning that this file should be copied from the user's home directory to the resource before the execution can begin. Under : : OUTPUTFILES : : a number of result files are listed, meaning that they should be copied to the user's home directory after job completion. The term +JOBID+ is a generic reference to the unique id of the job, which in this case is used to create a unique directory for each execution so that the result files do not interfere with each other. Finally $:$ : CPUTIME : : is set to $345600 \mathrm{~s}$, or $96 \mathrm{~h}$, to ensure that the job will have sufficient time to complete.

When we introduced the runtime environment it also greatly simplified the job specification file section. The Mutation Cluster jobs are set up and controlled in the tool using a number of input files, some of which are shared for all jobs and some of which are specific to the individual jobs. In the initial setup, all those files were specified as input files so that MiG automatically copied each input file to the job environment before execution. By using the runtime environment we could refactor the set of input files into a library of modules stored with the runtime environment, and a single job-specific file; after this, only the job-specific file is specified and thus copied for the job execution.

The experiment included too many jobs to manually dispatch and monitor through the web interface. Instead we downloaded

\begin{tabular}{cccc}
\hline & Execution time & Queue time & Total time \\
\hline Minimum & 0.1 & 0.0 & 2.1 \\
Average & 45.6 & 55.3 & 101.0 \\
Maximum & 392.4 & 504.9 & 677.7 \\
\hline
\end{tabular}

Fig. 3. Job flow statistics — all values are in hours.

the MiG executables and created simple scripts to automate some level of job management using those executables. The scripts are connected to a MySQL (http://www.mysql.com) database for keeping track of job parameters and job results. Since MiG automatically hands out jobs to resources when they are idle, large batches of jobs can be submitted to the MiG server without worrying about load balancing and congestion. During the experiment we introduced automatic resubmission of failed jobs in MiG so that we didn't have to worry about lost jobs due to limited resource failures, either.

All jobs were submitted at the same time and thus the first job started running almost immediately while the last jobs were queued for a long time. The resources that were made available to the GeneRecon experiments differed from $1.7 \mathrm{GHz}$ Intel Celeron CPUs to, $3.2 \mathrm{GHz}$ Intel Xeon-64 CPUs. This, combined with the varying execution times of the individual jobs, meant that total turnover times varied from a minimum $42 \mathrm{~s}$ to a maximum of more than 16 days! The minimum, maximum and average times for total turnover time, time in queue and time execution is shown in Fig. 3.

\subsection{Analysis results}

For the first part of the experiment we have validated that introducing the mutation and null cluster gives a runtime speedup, but does not reduce the method's ability to locate the disease locus significantly. For this study we have, for datasets simulated under various parameters, run several chains of the algorithm both with and without clustering, and then calculated the distance from the true location to the inferred location.

Fig. 4 shows the results for $\rho=400$, or $1 \mathrm{cM}$, for the disease model $1 / 0$ - all mutants being affected and no wild types being affected - for cluster sizes 100, 75, and 50. Little accuracy is lost when going from a mutation cluster size of 100 - corresponding to the original shattered coalescent model [8] - to a cluster size of 75 , whereas somewhat more is lost when going to a cluster size of 50 . That some accuracy is lost is, perhaps, not surprising considering that with this disease model all affected are mutants and none belong in the null cluster. For $\rho=40$, or $0.1 \mathrm{cM}$, the reduction in accuracy when moving from a mutation cluster size of 100 to 50 , still for the disease model 1/0, is much less, as shown in Fig. 5; except for the leftmost column in the figure, there is very little loss in accuracy. Varying the disease model to $0.8 / 0.1$ - that is, 

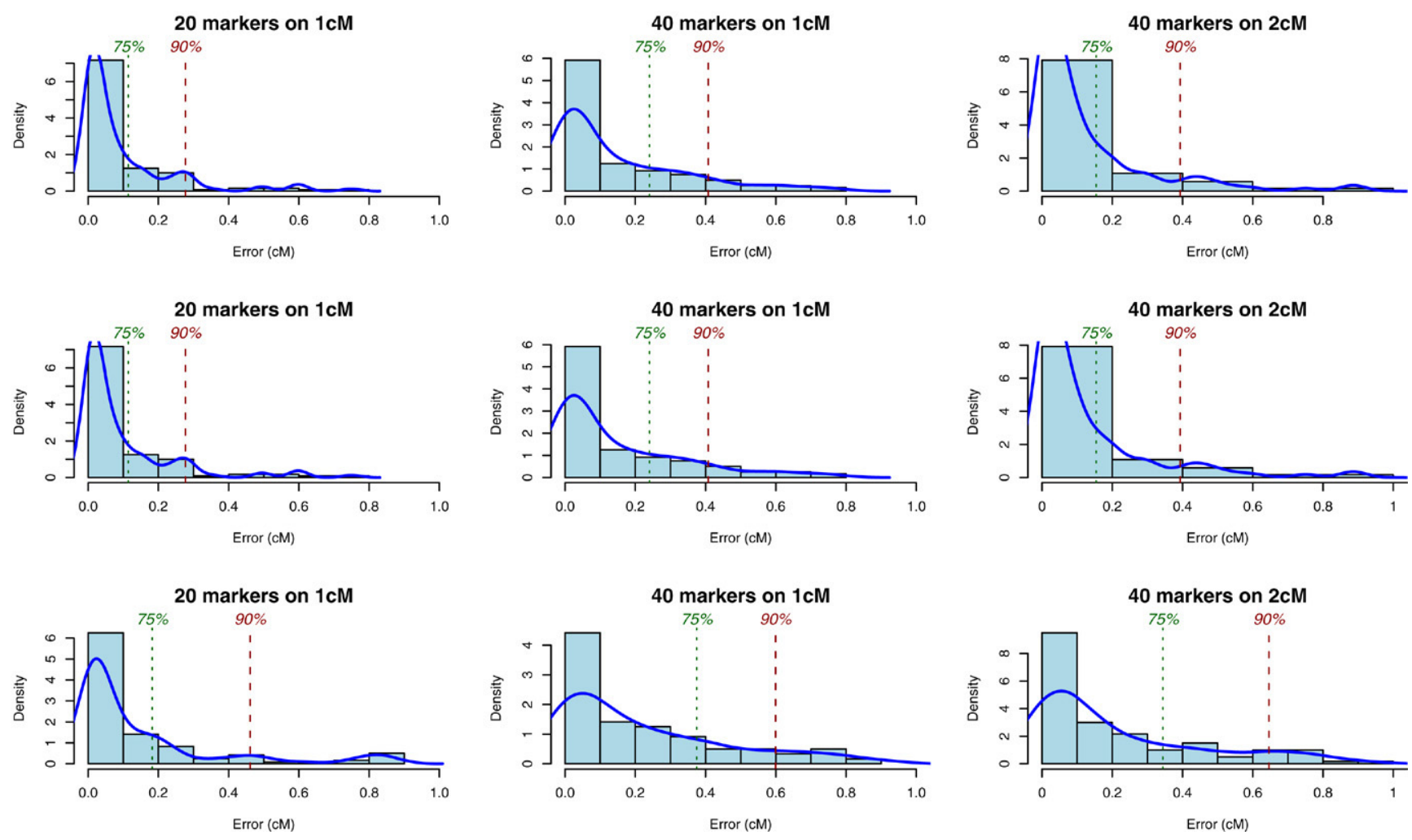

Fig. 4. Varying cluster sizes over a $1 \mathrm{cM}$ region. Disease model is $1 / 0$. The plots show the errors (distance from true disease locus to inferred disease locus) for varying mutation cluster sizes $(100,75,50$, from top to bottom, where 100 is the total number of affected) and varying marker densities: 20 markers on $1 \mathrm{cM}$ in the leftmost column, 40 markers in the middle column, and 40 markers spread over $2 \mathrm{cM}$ where the disease locus is in the middle $1 \mathrm{cM}$.
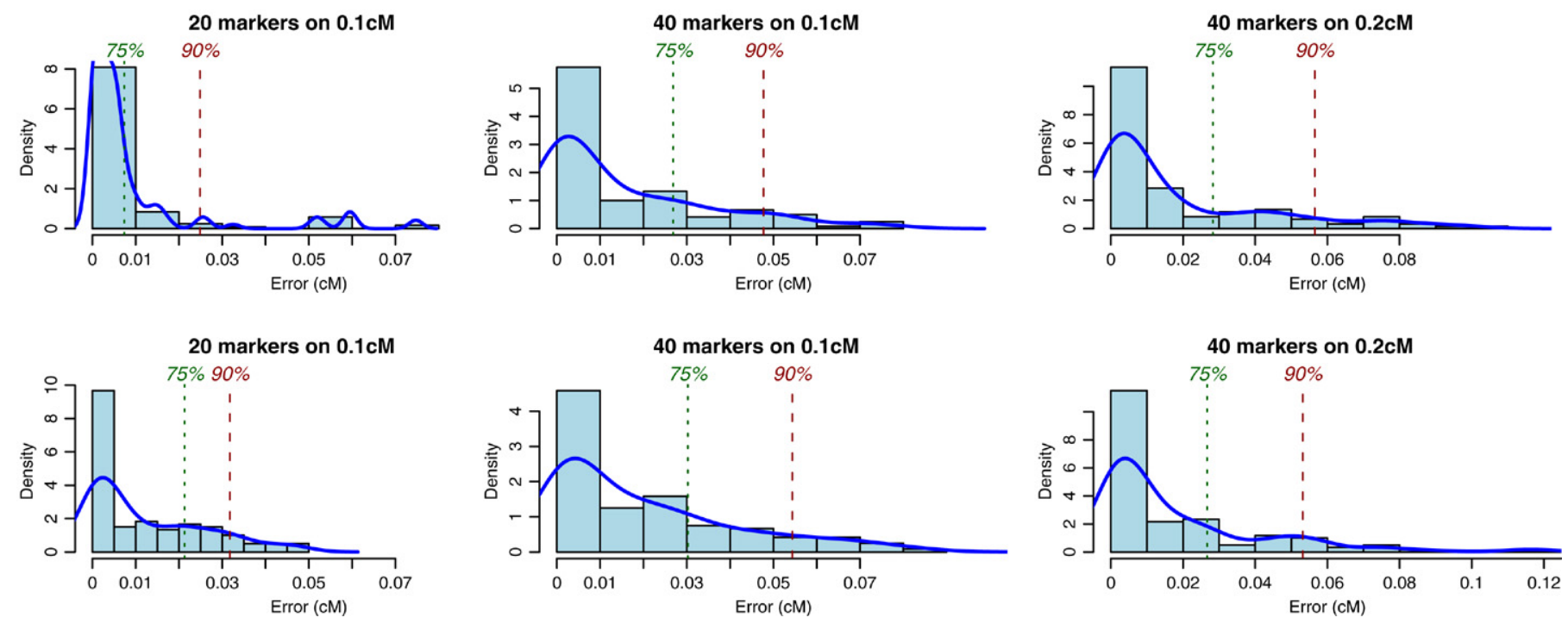

Fig. 5. Varying cluster sizes over a $0.1 \mathrm{cM}$ region. Disease model is $1 / 0$. The plots show the errors (distance from true disease locus to inferred disease locus) for varying mutation cluster sizes (100, 50, from top to bottom, where 100 is the total number of affected) and varying marker densities: 20 markers on $0.1 \mathrm{cM}$ in the leftmost column, 40 markers in the middle column, and 40 markers spread over $0.2 \mathrm{cM}$ where the disease locus is in the middle $0.1 \mathrm{cM}$.

mutants are affected with probability 0.8 and wild types with probability 0.1 - we see a small loss in accuracy for some of the settings for $\rho=400$, see Fig. 6, but interestingly the loss is smaller for the mutation cluster size 50 than for mutation cluster size 100. Still, cluster size 50 is less accurate than cluster size 100 for both disease models.
The analysis shows that there is some loss in accuracy when using a smaller mutation cluster size than the full set of affected individuals, but that this loss is not significant for a cluster size of 75 out of 100 for $\rho=400$ or 50 out of 100 for $\rho=40$. This despite that the running time is reduced from several days to one day or a quarter of a day - the exact measurement of 

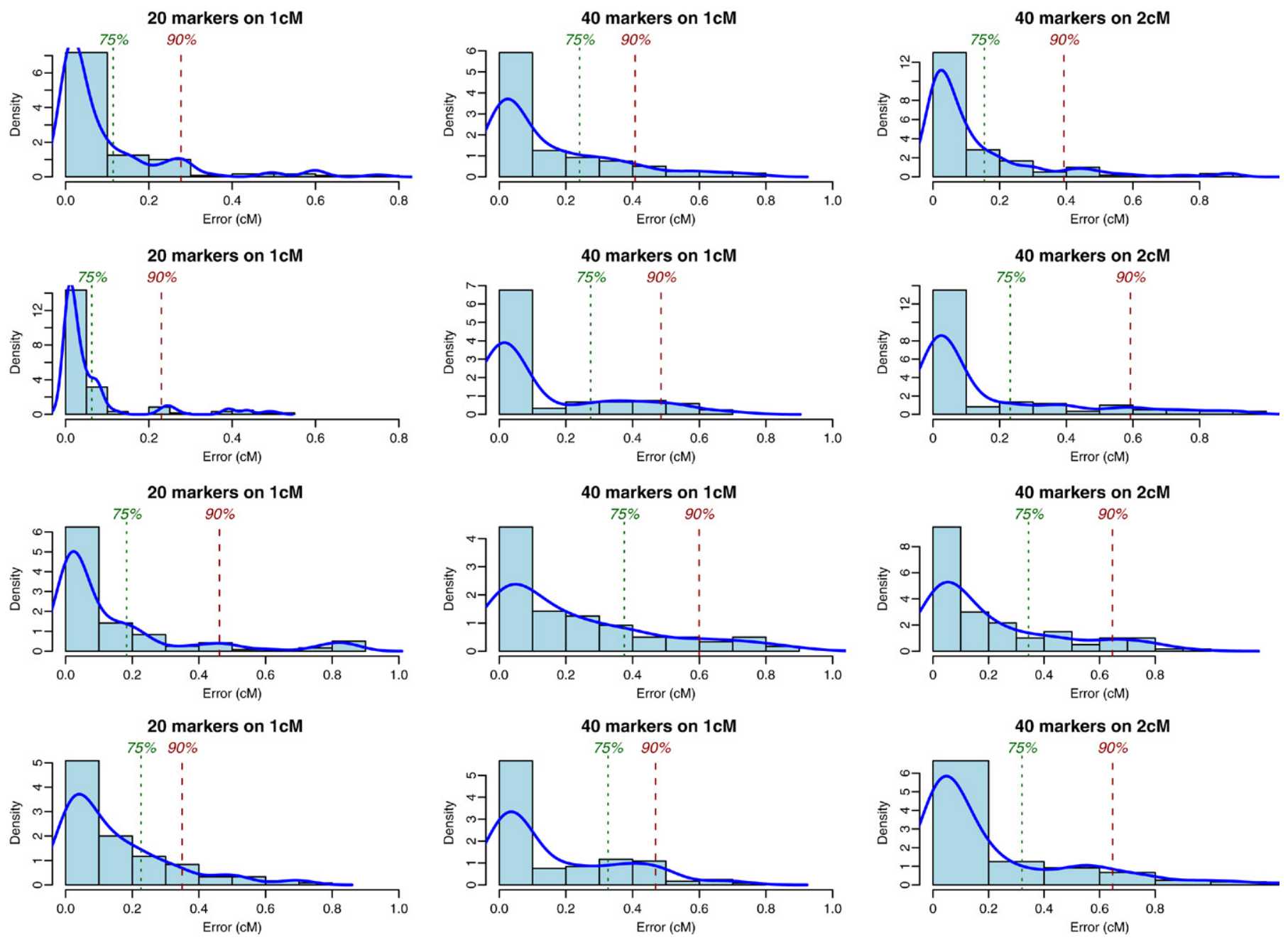

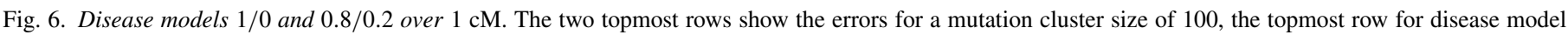
$1 / 0$, the second for disease model $0.8 / 0.2$; the next bottommost rows show the same setting but for a mutation cluster size of 50 .

the running time has not been done yet since the heterogeneous nature of $\mathrm{MiG}$ complicates this.

\section{Conclusions and future work}

The work presented in this paper represents early experiences with both Minimum intrusion Grid and using Grid for GeneRecon experiments. Nonetheless, we have successfully run a large scale bioinformatics Grid experiment with GeneRecon on MiG. In a period of a few weeks the project executed about five CPU-years of jobs. Grid or Public Resource Computing (PRC) solutions are obvious targets when taking on parallelizable projects with such an amount of required CPU power. Porting a custom application to run on large scale using Grid additionally turned out to be a very easy task with the Minimum intrusion Grid. In short we only needed to create a static version of the executable and produce a script that transforms our large parameter sweep into a number of individual Grid jobs, each of which are created by filling in a simple job template. In the future we would like to also take on experiments that are less parallel by nature, in order to emphasise that MiG works well with applications that are more suited to Grid than to PRC, too.
The stability of MiG and the strength of the automatic resubmission of failed jobs were emphasised by our experiment where thousands of jobs were submitted at the same time just before a holiday and left unattended until they had all completed, roughly a month later. It should also be pointed out that running the experiment on $\mathrm{MiG}$ did not add considerable overhead. In addition to providing job execution facilities, MiG natively supports virtual organisations through the VGrid features. This allowed the bioinformatics group behind the GeneRecon project to easily exchange files and computational resources with the MiG group while the groups were at different universities and with hundreds of kilometres between them.

Our experiments with GeneRecon on MiG have been rewarding and we plan to continue this work in several directions. From a bioinformatics perspective there are many topics that become feasible to explore with the availability of large computational resources, such as those provided by MiG. One direction we wish to explore with the Mutation Cluster method is to extract information about how long each case has been included in the mutation cluster, as opposed to the null cluster, and see if we can infer from this which cases are mutants and which cases are diseased 
from environmental effects. The efficiency of the disease loci inferences depends very much on the data set which in turn depends on various parameters, such as recombination and mutation rates, population structure, and penetrance. Without access to massive CPU resources, this parameter space cannot be explored to fully figure out which parameters significantly affect the inference results and which have little effect on the efficiency of the algorithms.

An interesting problem, from the Grid point of view, that will be further investigated, is whether it is possible to use check-pointing techniques to support migration of GeneRecon processes. This has become increasingly interesting with the recent introduction of support for utilising 'Screen Saver Science' as part of the MiG resource model. If a large number of computers join MiG when they are in screen-saver mode, the amount of available compute-resources increases significantly, but it is unlikely that a computer will remain in screen-saver mode for long enough to complete an average GeneRecon job. If jobs can be migrated or suspended for resumed execution later, even relatively short term resources may prove far more valuable.

\section{References}

[1] I. Foster, A new infrastructure for 21 st century science, Physics Today 55 (2) (2002) 42-47.

[2] H.H. Karlsen, B. Vinter, VGrids as an implementation of virtual organizations in Grid computing, in: Emerging Technologies for Nextgeneration GRID 2006, 2006 (in press).

[3] L.C. Lazzeroni, A chronology of fine-scale gene mapping by linkage disequilibrium, Statistical Methods in Medical Research 10 (2001) 57-76.

[4] J.S. Liu, C. Sabatti, J. Teng, B.J.B. Keats, N. Risch, Bayesian analysis of haplotypes for linkate disequilibrium mapping, Genome Research 11 (2001) 1716-1724.

[5] T. Mailund, M. Schierup, C. Pedersen, P. Mechlenborg, J. Madsen, L. Schauser, CoaSim: A flexible environment for simulating genetic data under coalescent models, BMC Bioinformatics 6 (252) (2005).

[6] T. Mailund, M. Schierup, C. Pedersen, J. Madsen, J. Hein, L. Schauser, GeneRecon-A coalescent based tool for fine-scale association mapping, Bioinformatics (2006), doi:10.1093/bioinformatics/btl153. Advance access published online.

[7] J. Molitor, P. Marjoram, D. Thomas, Fine-scale mapping of disease genes with multiple mutations via spatial clustering techniques, American Journal of Human Genetics 73 (2003) 1368-1384.

[8] A.P. Morris, J.C. Whittaker, D.J. Balding, Fine-scale mapping of disease loci via shattered coalescent modeling of genealogies, American Journal of Human Genetics 70 (2002) 686-707.
[9] D.E. Reich, E.S. Lander, On the allelic spectrum of human disease, Trends in Genetics 17 (9) (2001) 502-510.

[10] B. Vinter, The architecture of the minimum intrusion Grid (MiG), Communicating Process Architectures 2005 (2005) 189-201.

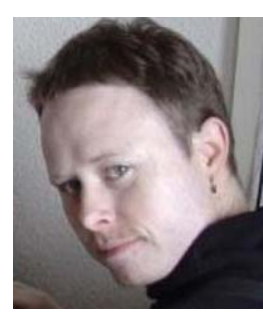

Thomas Mailund is a post.doc. at the Department of Statistics, University of Oxford, UK. He received his $\mathrm{Ph} . \mathrm{D}$. from the Department of Computer Science at the University of Aarhus, Denmark, in 2003, and worked as an assistant professor at Bioinformatics Research Center, University of Aarhus, 2003-2006.

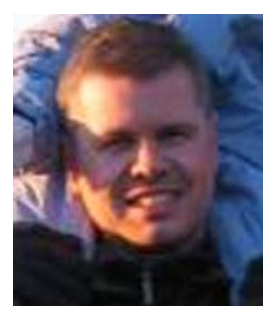

Christian N. S. Pedersen is an Associate Professor at the Department of Computer Science (DAIMI), University of Aarhus, Denmark and director of the Bioinformatics Research Center (BiRC). Furthermore he is affiliated to the Center for Basic Research in Computer Science (BRICS). Christian got his Ph.D. in Computer Science from the University of Aarhus, Denmark in 1999.

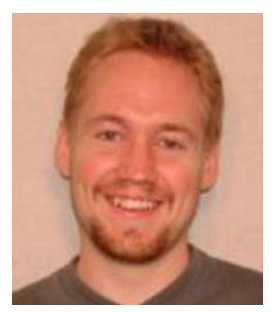

Jonas Bardino is a research assistant at the Department of Computer Science, University of Copenhagen, Denmark. He received his Master of Computer Science from the University of Southern Denmark, Odense, Denmark in 2004.

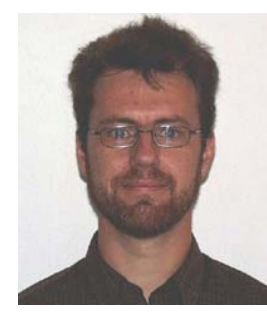

Brian Vinter got his MS.CE from Aalborg University, 1994 and Dr. Scient from Tromsoe University, 1999. Dr. Vinter is Professor of Computer Science at the University of Copenhagen, within supercomputing and Grid computing. He is the former director of the Nordic DataGrid Facility.

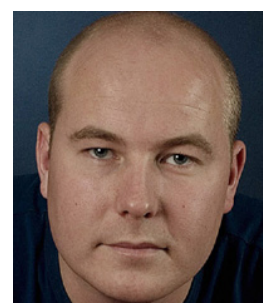

Henrik H. Karlsen got his Master of Science in Computer Science from the University of Southern Denmark in 2005. Currently he is employed as a research assistant at University of Copenhagen with primary focus within Distributed and Grid computing. 